\title{
Minimal Depression: How Does It Relate to Upper-Extremity Impairment and Function in Stroke?
}

\author{
Lindy L. Weaver, Stephen J. Page, Lynne Sheffler, John Chae
}

MeSH TERMS

- depression

- motor skills

- outcome assessment (health care)

- stroke

- upper extremity
OBJECTIVE. We sought to determine the association between minimal depression, upper-extremity (UE) impairment, and UE motor function in a cohort of participants with subacute stroke.

METHOD. We conducted a retrospective, secondary analysis of an interventional study. Correlational analyses were performed using the following outcome measures: the UE section of the Fugl-Meyer Assessment (FM), the functional ability section of the Arm Motor Ability Test (AMAT), and the Beck Depression Inventory (BDI-II).

RESULTS. We found a negative correlation between BDI-II and both the FM $(-.120, p=.196)$ and the AMAT $(-.110, p=.275)$; however, this correlation was not statistically significant. Women exhibited higher depression scores $(8.75 \pm 0.78)$ than men $(6.29 \pm 0.46 ; p=.008)$.

CONCLUSION. Low levels of depression are not associated with UE motor impairment and function in people with minimal to moderate UE disability levels. Poststroke depression occurs more frequently in women, warranting additional research on sex-specific differences. Given the proliferation of UE therapies targeting this group, this information is important for effective therapy planning and implementation.

Weaver, L. L., Page, S. J., Sheffler, L., \& Chae, J. (2013). Minimal depression: How does it relate to upper-extremity impairment and function in stroke? American Journal of Occupational Therapy, 67, 550-555. http://dx.doi.org/ 10.5014/ajot.2013.008391

Doststroke depression (PSD) is the most common neuropsychiatric disorder exhibited after stroke, affecting about a third of the growing stroke population (Altieri et al., 2012; Chemerinski \& Robinson, 2000; Roger et al., 2012). Even low levels of PSD among patients with stroke are posited to coincide with diminished levels of function (Carod-Artal, Egido, González, \& Varela de Seijas, 2000; Ellis, Zhao, \& Egede, 2010; House, Knapp, Bamford, \& Vail, 2001; Pohjasvaara, Vataja, Leppävuori, Kaste, \& Erkinjuntti, 2001). For example, assistance needed to perform daily activities and length of stay were each correlated with PSD in acutely hospitalized stroke survivors, even when PSD levels were minimal to moderate (Carney \& Freedland, 2002; Gillen, Tennen, McKee, Gernert-Dott, \& Affleck, 2001; Roger et al., 2012).

Although research has contributed to our understanding of PSD, past studies have been limited in that they tested PSD once, during the acute phase. Most stroke survivors experience ongoing spontaneous recovery during the acute phase, and unless researchers test for PSD in the subacute stages and beyond, their ability to identify PSD as a stroke outcome is limited. Likewise, the evolving nature of deficits during the acute phase makes it difficult to determine whether PSD constitutes a substantive stroke outcome or merely an artifact of the adjustment process to new deficits. Use of generalized measures of physical dependence that allow for compensatory strategies (e.g., the FIM ${ }^{\mathrm{TM}}$, the modified Rankin Scale, the Barthel Scale; Gillen et al., 2001; Morrison, Pollard, Johnston, \& MacWalter, 2005; Pohjasvaara et al., 2001; Snaphaan, van der Werf, Kanselaar, \& de Leeuw, 2009) also limits the ability to determine how PSD relates to 
specific motor impairments or functional limitations specific to the hemiparetic upper limb. Enrollment of convenience samples of hospitalized stroke patients is also susceptible to sample bias depending on the number of clients, location, and populations that a particular hospital serves. Such bias can yield study samples that are not well distributed across demographic variables and may even exclude certain subgroups altogether.

Given these shortfalls and the fact that upper-extremity (UE) hemiparesis is the most common and disabling of stroke sequelae, the need remains to determine the association between PSD and UE motor impairment and UE functional ability using stroke-specific measures and large, well-defined, community-based samples. Because occupational therapists focus on reducing the severity of psychosocial impairments and their functional impact, measurement of these sequelae is fundamental to costeffective, appropriate treatment planning.

Considering the limitations of previous research, the purposes of this study were to determine the association of minimal depression with UE motor impairment and with UE motor function in patients with subacute stroke. We recruited participants in the current study using various methods to obtain a random sample that was representative of the intended study population. Several motor therapy regimens have shown efficacy in patients with stroke exhibiting minimal to moderate UE impairment (De Kroon, Ijzerman, Lankhorst, \& Zilvold, 2004; Page, Levine, \& Leonard, 2007), yet little is known about PSD's impact on this subgroup. Additionally, although it is known that high PSD levels are likely to influence impairment and function, low levels of depression may also subtly influence motor impairment outcomes but have not heretofore been examined. Thus, to better characterize these populations, we further delimited the sample to people with minimal levels of PSD (as characterized by a score $\leq 19$ on the Beck Depression Inventory, Second Edition [BDI-II]; Beck, Steer, \& Carbin, 1988) and minimal to moderate UE impairment. We hypothesized that higher scores on the Fugl-Meyer Assessment (FM; Fugl-Meyer, Jääskö, Leyman, Olsson, $\&$ Steglind, 1975) would be correlated with lower scores on the BDI-II and that higher scores on the Arm Motor Ability Test (AMAT; Kopp et al., 1997) would be correlated with lower BDI-II scores. To our knowledge, this was the first study testing the associations between PSD and UE impairment and UE functional limitation using stroke-specific movement measures.

The current study was expected to be of particular interest to occupational therapy practitioners given that even low levels of depression are thought to compromise motivation, concentration, and problem-solving ability (Bonder, 2004), which can undermine volition and independent activity participation and performance. In addition, occupational therapy practitioners often specialize in the treatment and management of the UE, frequently assess people with depression, and often integrate strategies into their treatments that address both PSD and UE symptoms.

\section{Method}

\section{Study Design}

This study was a secondary analysis of data gathered as part of a randomized controlled trial testing electrical stimulation for paretic UE movement in subacute stroke. As part of this three-center study, a battery of screening measures (including the BDI-II) and paretic UE motor outcome measures (including the FM and AMAT) were administered before and after the 10 -wk intervention period. The measures were administered within 2 wk of study enrollment by a single rater at each site in a quiet room adjacent to where the study treatments were taking place. Equipment for testing (e.g., height of tables and seats at which participants sat for the AMAT, items used) and testing procedures at each site were held consistent.

Each rater underwent initial training on administration of the measures and participated in periodic inservices to ensure that test administration was consistent across study sites. The current study focuses on analysis of scores obtained for the BDI-II, FM, and AMAT during the pretesting study phase before any intervention had occurred. After pretesting, participants were randomized to various intervention conditions; participation in these interventions could have affected UE impairment, UE function, and depression levels to varying extents. Thus, we examined these relationships before any intervention took place. We obtained institutional review board approval at each site before conducting the study.

\section{Participants}

We recruited volunteers for the trial from across the midwestern United States using several strategies, including newspaper advertisements, other print advertisements (e.g., pamphlets) placed in rehabilitation clinics and stroke support groups near enrolling sites, and "lunchand-learns" provided by study team members to local rehabilitation clinicians. As volunteers came forward, we applied several screening criteria. Inclusion criteria were as follows: (1) within 6 mo of first clinical hemorrhagic or nonhemorrhagic stroke, (2) ability to produce any wrist 
extension with manual muscle grade $\leq 4 / 5$ on the Medical Research Council scale (Gregson et al., 2000), (3) presence of volitionally activated electromyography signal from the paretic extensor carpi radialis (ECR) of at least $5 \mu \mathrm{V}$ in amplitude, (4) age 18-94 yr, and (5) demonstration of tolerance to surface transcutaneous neuromuscular electrical stimulation of the ECR and extensor digitorum communis. Exclusion criteria were as follows: (1) insensate or edematous skin; (2) history of cardiac arrhythmia, demand pacemakers, or any other implanted electronic system; (3) pregnancy or uncontrolled seizure disorder; (4) scores below the fourth quartile on ageadjusted norms on the Mini-Mental State Examination (Folstein, Folstein, \& McHugh, 1975); and (5) uncompensated hemineglect, moderate to severe depression, ipsilateral lower-limb motor neuron lesion, Parkinson's disease, spinal cord injury, traumatic brain injury, multiple sclerosis, or wrist and finger contracture.

\section{Assessments}

The upper-extremity section of the FM was the primary outcome measure in this study. FM items evaluate specific upper-limb movements from proximal (e.g., shoulder abduction, internal rotation) to distal (e.g., mass grasp, pincer grasp). Each test item is scored on a 3-point ordinal scale $(0=$ cannot perform to $2=$ can perform fully $)$ and summed to provide a maximum score of 66 . The FM has been shown to have high test-retest reliability (total = $.98-.99$, subtests $=.87-1.00$ ), interrater reliability, and construct validity (Duncan, Propst, \& Nelson, 1983).

To evaluate the impact of change in paretic UE impairment on upper-limb-specific activity limitations, we used the AMAT as a secondary outcome measure. The AMAT is a 13-item test in which ability to perform common activities of daily living (e.g., using a knife and fork, eating with a spoon, combing hair, tying shoelaces) is assessed according to three scales: (1) a Functional Ability scale that examines extent of affected UE use $(0=$ does not perform with affected $U E$ to $5=$ does use $U E$ at a level comparable to unaffected side); (2) a Quality of Movement scale $(0=$ no movement initiated to $5=$ normal movement); and (3) time in seconds to perform each of the 13 items, with time beginning at movement initiation and ending on movement completion. The AMAT demonstrates high interrater reliability (Spearman correlation $=.97-.99$ ) and internal consistency (Cronbach's $\alpha=.93-.99)$ and satisfactory concurrent validity (Cronbach's $\alpha=.62$; Kopp et al., 1997).

To measure poststroke depression, we used the BDI-II. The BDI-II comprises 21 self-report multiple-choice questions. Test items are aligned with the Diagnostic and Statistical Manual of Mental Disorders, Fourth Edition (American Psychiatric Association, 1994) criteria for depression. Each question lists four statements related to a depressive symptom, and answers are scored from 0-3, with higher scores indicating increased symptom severity. Total test scores indicate the following depression levels: 0-13 = minimal depression, 14-19= mild depression, 20-28 = moderate depression, and 29-63 = severe depression. Patients with scores $\geq 19$ on the BDI-II were excluded given the focus of this study and the overall trial. The BDI-II has demonstrated high internal reliability $(\alpha=.92)$ and sensitivity $(84 \%)$ and strong convergent and discriminant validity (Dozois, Dobson, \& Ahnberg, 1998; Sprinkle et al., 2002). In addition, as with other stroke rehabilitation trials, we recorded demographic variables including age, time (mo) poststroke, and stroke location and type.

\section{Data Analyses}

All data analyses were performed using IBM SPSS (Version 19.0; IBM Corporation, Armonk, NY). Participants' demographic characteristics were analyzed using means, standard deviations, and ranges. Descriptive statistics for baseline scores of depression and motor performance were also produced. This study aimed to identify the association between preintervention scores on the BDIII, FM, and AMAT. Thus, a correlational analysis of each continuous dependent variable was performed. The dependent variables were preintervention FM and AMAT scores. The independent variable was preintervention BDI-II scores. A nonparametric Spearman correlation was used, given the distribution of these data. This test is robust to violations of normality and less affected by outliers (Chen \& Popovich, 2002).

\section{Results}

\section{Baseline Characteristics}

The full sample included 122 participants. Four participants were removed from analysis because of missing data ( 1 from the FM, 1 from the BDI-II, and 2 from the AMAT; Table 1). The analyzed sample included the remaining 118 participants. The baseline demographic

\section{Table 1. Assessment Score Means and Standard Errors}

\begin{tabular}{lcc}
\hline Assessment & $n$ & $M(S E)$ \\
\hline Fugl-Meyer Assessment & 121 & $26.78(1.14)$ \\
Beck Depression Inventory, Second Edition & 121 & $7.45(0.44)$ \\
Arm Motor Activity Test & 120 & $1.69(0.1)$ \\
\hline
\end{tabular}

Note. $M=$ mean; $S E=$ standard error. 
statistics for the sample are presented in Table 2. The number of days from stroke onset until participation in the study varied from 12 to 183 days, with an average of 91.8 days \pm 4.62 days. Slightly more than half of the participants (58.2\%) were men. Lesion side was relatively evenly distributed: $48.4 \%$ of participants had right-side lesions and $51.6 \%$ had left-side lesions.

\section{Outcomes}

Independent-sample $t$ tests were performed to determine whether significant differences in AMAT, FM, and BDI-II scores were observed for various demographic groups (Table 3 ). The BDI-II scores were significantly higher for female participants $(8.75 \pm 0.78)$ than for male participants $(6.29 \pm 0.46 ; p=.008)$. No significant differences in AMAT, FMA, or BDI-II scores were noted for the variables of lesion side, stroke type, and right or left hemiparesis. The remaining demographic variables, such as other types of stroke, were not tested because of the significant imbalance in sample size of participants with and without the characteristics.

The correlation between AMAT and BDI-II scores was $-.101(p=.275)$. The correlation between FMA and BDI-II scores was $-.120(p=.196)$. Both correlations were negative but not statistically different from zero at a Bonferroni-adjusted $\alpha$ level of .025 (Table 4).

\section{Table 2. Sample Characteristics}

\begin{tabular}{lr}
\hline Characteristic & $n(\%)$ \\
\hline Race & $84(68.9)$ \\
Caucasian & $33(27.0)$ \\
African American & $2(1.6)$ \\
Hispanic/Latino & $2(1.6)$ \\
Asian/Pacific Islander & $1(0.8)$ \\
More than one race & \\
Gender & $71(58.2)$ \\
Male & $51(41.8)$ \\
Female & \\
Lesion side & $59(48.4)$ \\
Right & $63(51.6)$ \\
Left & \\
Hemiparesis & $60(49.2)$ \\
Right & $62(50.8)$ \\
Left & \\
Deficits & $5(4.9)$ \\
Apraxia & $14(11.5)$ \\
Dysphagia & $14(11.5)$ \\
Neglect & $23(18.9)$ \\
Cognition & $25(20.5)$ \\
Aphasia & $10(9.0)$ \\
Sensation & $7(5.7)$ \\
Bladder/bowel & $24(19.7)$ \\
Vision &
\end{tabular}

Note. $N=122$
Table 3. Assessment Score Means and Standard Errors, by Subgroup $(N=118)$

\begin{tabular}{|c|c|c|c|}
\hline Subgroup & $\begin{array}{c}\mathrm{FM}, \\
M(S E)\end{array}$ & $\begin{array}{l}\text { BDI-II, } \\
M(S E)\end{array}$ & $\begin{array}{l}\text { AMAT, } \\
M(S E)\end{array}$ \\
\hline \multicolumn{4}{|l|}{ Gender } \\
\hline Male & $26.9(1.5)$ & $6.3^{*}(0.5)$ & $1.7(0.1)$ \\
\hline Female & $27.2(1.9)$ & $8.8 \quad(0.8)$ & $1.7(0.2)$ \\
\hline \multicolumn{4}{|l|}{ Lesion side } \\
\hline Right & $25.4(1.6)$ & $7.2(0.6)$ & $1.5(0.1)$ \\
\hline Left & $28.5(1.6)$ & $7.4 \quad(0.6)$ & $1.9(0.1)$ \\
\hline \multicolumn{4}{|l|}{ Stroke type } \\
\hline Subarachnoid hemorrhage & $32.3(2.6)$ & $7.5 \quad(0.9)$ & $2.1(0.2)$ \\
\hline Arteriovenous malformation & $25.8(1.3)$ & $7.2(0.5)$ & $1.6(0.1)$ \\
\hline \multicolumn{4}{|l|}{ Paresis side } \\
\hline Right & $28.7(1.6)$ & $7.4 \quad(0.6)$ & $1.9(0.1)$ \\
\hline Left & $25.3(1.6)$ & $7.2(0.6)$ & $1.5(0.1)$ \\
\hline
\end{tabular}

Note. Independent-sample $t$ tests were used for analyses. AMAT = Arm Motor Activity Test; BDI-II = Beck Depression Inventory, 2nd Edition; FM = FuglMeyer Assessment; $M=$ mean; $S E=$ standard error.

${ }^{*} p=.008$.

\section{Discussion}

PSD is present in approximately one-third of stroke survivors and is thought to negatively influence functional outcomes. The limitations of studies to date have included heterogeneous samples, data collection only during the period of acute spontaneous recovery, and limited use of stroke-specific measures of UE impairment and function. The current study overcomes these limitations by determining the association between baseline motor performance scores and depression indexes using a large, well-defined sample of adults with stable motor deficits who exhibited minimal depression. This investigation provides insight into how PSD may affect treatment and recovery among stroke survivors exhibiting minimal to moderate UE impairments.

Contrary to the primary study hypothesis, higher motor-function scores on the FM and AMAT were not correlated with lower depression scores on the BDI-II. Thus, the analyses did not indicate that participants who had better motor function reported fewer depressive symptoms or vice versa. Even when stratifying the sample by BDI-II scores $(0-13, \geq 14)$, no significant relationships between higher FM and AMAT scores and lower levels of depression were observed.

Correlations may be difficult to detect in a sample of subacute, moderately impaired patients. It may also be that these patients are likely to experience additional recovery or that their daily lives are less affected by loss of function, causing them to adjust more readily to life after stroke and to experience fewer depressive symptoms. Another plausible explanation is that PSD does not affect people who are moderately impaired in the same way it 
Table 4. Assessment Score Correlations

\begin{tabular}{lccc}
\hline Measure & 1 & 2 & 3 \\
\hline 1. FM & - & $-.120(p=.196)$ & $.837(p=.000)$ \\
2. BDI-II & $-.120(p=.196)$ & - & $-.101(p=.275)$ \\
3. AMAT & $.837(p=.000)$ & $-.101(p=.275)$ & - \\
\hline
\end{tabular}

Note. Spearman rank order correlation (2-tailed) was used for analysis. AMAT= Arm Motor Activity Test; BDI-II = Beck Depression Inventory, 2nd Edition; $\mathrm{FM}=$ Fugl-Meyer Assessment.

affects those with more severe impairments, such that PSD presents less detriment to moderately impaired people's overall rehabilitation potential and recovery trajectory. Finally, the AMAT is a laboratory-based functional capacity measure and may not reflect actual performance in the community. Thus, we recommend that occupational therapy practitioners working closely with this population undertake accurate assessment and close monitoring of severity of depression. Using treatment strategies and interpersonal skills that facilitate patients' motivation, concentration, and problem-solving ability may also support their psychosocial needs.

Although secondary to the study purpose, PSD trends were noticeably different according to gender. Analyses showed that women demonstrated significantly $(p=.008)$ higher depression scores than men. This finding is consistent with the prevalence of depression in the general population and among women who have experienced stroke (Marcus et al., 2005; Poynter et al., 2009). In the general population, women are nearly twice as likely to experience an episode of major depression (Halbreich \& Kahn, 2007; Poynter et al., 2009). Following stroke, the prevalence of PSD is markedly higher among women than men (Poynter et al., 2009). Reported differences in stroke outcomes for women following their first stroke may contribute to this difference (Mackay \& Mensah, 2004). For example, after a first stroke, women remain in the hospital longer and have more disabilities than men receiving similar care (Mackay \& Mensah, 2004). For occupational therapy professionals, the knowledge that women are at greater risk for PSD can be useful when selecting assessments and designing individualized treatments.

\section{Implications for Occupational Therapy Practice}

- For people experiencing moderate poststroke disability, PSD may not be associated with level of UE motor impairment or function.

- Accurate assessment and close monitoring of severity of depression are recommended throughout the evaluation and treatment processes.
- Using treatment strategies and interpersonal skills that facilitate a patient's motivation, concentration, and problem-solving ability may support their psychosocial needs.

- Women's greater risk for PSD should be considered when selecting assessments and designing individualized treatments.

\section{Study Limitations and Future Directions}

As a secondary analysis, this study has inherent limitations. PSD was not the primary focus of the original study. The BDI-II was implemented as a screening tool and was the only measurement of depressive symptoms used. Use of multiple measures of depression and even personal surveys may make it easier to detect or quantify the relationship between minimal PSD and motor performance. An additional limitation is that participants had to meet strict inclusion criteria regarding level of motor function and deficit. Few participants displayed deficits in commonly affected areas such as vision, sensation, cognition, or bowel and bladder control. Thus, our sample of mildly to moderately impaired patients with minimal PSD may have been too restrictive and may explain the weak relationship between motor function scores and depression indexes in this study. Use of stroke-specific measures was an asset in this study because such measures are valuable for characterizing motor impairment and its relationship to functional tasks.

We see this study as an iterative first step toward a better understanding of PSD and motor function after stroke; our goal is to expand the study to participants with increased depression and impairment. Looking at people with moderate depression and mild or moderate UE impairment may yield more information on the interplay between varying levels of PSD and UE impairment and function. As a result of this study, we recommend that future research focus on three areas: (1) clinical samples with a wider range of depression scores to provide insight into various depression levels and corresponding impairment and functional outcomes, (2) the continued use of stroke-specific measures of UE motor impairment and UE functional ability, and (3) sex-specific differences in PSD among stroke survivors.

\section{Acknowledgments}

This work was supported by Grant R01HD49777 from the National Center for Medical Rehabilitation Research of the National Institute for Child Health and Human Development, Grant R01AT004454-04 from the National Institutes of Complementary and Alternative Medicine, and Grant 1UL1RR024989 from the National Center for Research Resources. 


\section{References}

Altieri, M., Maestrini, I., Mercurio, A., Troisi, P., Sgarlata, E., Rea, V., . . . Lenzi, G. L. (2012). Depression after minor stroke: Prevalence and predictors. European Journal of Neurology, 19, 517-521. http://dx.doi.org/10.1111/j.1468-1331. 2011.03583.x

American Psychiatric Association. (1994). Diagnostic and statistical manual of mental disorders (4th ed.). Washington, DC: Author.

Beck, A. T., Steer, R. A., \& Carbin, M. G. (1988). Psychometric properties of the Beck Depression Inventory: Twenty-five years of evaluation. Clinical Psychology Review, 8, 77-100. http://dx.doi.org/10.1016/0272-7358(88)90050-5

Bonder, B. (2004).Psychopathology and function. Thorofare, NJ: Slack.

Carney, R. M., \& Freedland, K. E. (2002). Psychological distress as a risk factor for stroke-related mortality. Stroke, 33, 5-6.

Carod-Artal, J., Egido, J. A., González, J. L., \& Varela de Seijas, E. (2000). Quality of life among stroke survivors evaluated 1 year after stroke: Experience of a stroke unit. Stroke, 31, 2995-3000. http://dx.doi.org/10.1161/01. STR.31.12.2995

Chemerinski, E., \& Robinson, R. G. (2000). The neuropsychiatry of stroke. Psychosomatics, 41, 5-14. http://dx.doi. org/10.1016/S0033-3182(00)71168-6

Chen, P. Y., \& Popovich, P. M. (2002). Correlation: Parametric and nonparametric measures. Thousands Oaks, CA: Sage.

De Kroon, J., Ijzerman, M., Lankhorst, G., \& Zilvold, G. (2004). Electrical stimulation of the upper limb in stroke: Stimulation of the extensors of the hand vs. alternate stimulation of flexors and extensors. American Journal of Physical Medicine and Rehabilitation, 83, 592-600.

Dozois, D. J. A., Dobson, K. S., \& Ahnberg, J. L. (1998). A psychometric evaluation of the Beck Depression Inventory-II. Psychological Assessment, 10, 83-89. http://dx. doi.org/10.1037/1040-3590.10.2.83

Duncan, P. W., Propst, M., \& Nelson, S. G. (1983). Reliability of the Fugl-Meyer assessment of sensorimotor recovery following cerebrovascular accident. Physical Therapy, 63, 1606-1610.

Ellis, C., Zhao, Y., \& Egede, L. E. (2010). Depression and increased risk of death in adults with stroke. Journal of Psychosomatic Research, 68, 545-551. http://dx.doi.org/ 10.1016/j.jpsychores.2009.11.006

Folstein, M., Folstein, S., \& McHugh, P. (1975). "MiniMental State": A practical method for grading the cognitive state of patients for the clinician. Journal of Psychiatric Research, 12, 189-198.

Fugl-Meyer, A. R., Jääskö, L., Leyman, I., Olsson, S., \& Steglind, S. (1975). The post-stroke hemiplegic patient: 1. A method for evaluation of physical performance. Scandinavian Journal of Rehabilitation Medicine, 7, 13-31.

Gillen, R., Tennen, H., McKee, T. E., Gernert-Dott, P., \& Affleck, G. (2001). Depressive symptoms and history of depression predict rehabilitation efficiency in stroke patients. Archives of Physical Medicine and Rehabilitation, 82, 1645-1649. http://dx.doi.org/10.1053/apmr.2001.26249

Gregson, J., Leathley, M., Moore, A., Smith, T., Sharma, A., \& Watkins, C. (2000). Reliability of measurements of muscle tone and muscle power in stroke patients. Age and Ageing, 29, 223-228.

Halbreich, U., \& Kahn, L. S. (2007). Atypical depression, somatic depression and anxious depression in women: Are they gender-preferred phenotypes? Journal of Affective Disorders, 102, 245-258. http://dx.doi.org/10.1016/j. jad.2006.09.023

House, A., Knapp, P., Bamford, J., \& Vail, A. (2001). Mortality at 12 and 24 months after stroke may be associated with depressive symptoms at 1 month. Stroke, 32, 696-701. http://dx.doi.org/10.1161/01.STR.32.3.696

Kopp, B., Kunkel, A., Flor, H., Platz, T., Rose, U., Mauritz, K. H., . . Taub, E. (1997). The Arm Motor Ability Test: Reliability, validity, and sensitivity to change of an instrument for assessing disabilities in activities of daily living. Archives of Physical and Medical Rehabililitation, 78, 615-620. http://dx. doi.org/10.1016/S0003-9993(97)90427-5

Mackay, J., \& Mensah, G. A. (2004). The atlas of heart disease and stroke. Geneva: World Health Organization.

Marcus, S. M., Young, E. A., Kerber, K. B., Kornstein, S., Farabaugh, A. H., Mitchell, J., . . . Rush, A. J. (2005). Gender differences in depression: Findings from the STAR*D study. Journal of Affective Disorders, 87, 141-150. http://dx.doi.org/10.1016/j.jad.2004.09.008

Morrison, V., Pollard, B., Johnston, M., \& MacWalter, R. (2005). Anxiety and depression 3 years following stroke: Demographic, clinical, and psychological predictors. Journal of Psychosomatic Research, 59, 209-213. http://dx.doi. org/10.1016/j.jpsychores.2005.02.019

Page, S. J., Levine, P., \& Leonard, A. (2007). Mental practice in chronic stroke: Results of a randomized, placebo-controlled trial. Stroke, 38, 1293-1297. http://dx.doi.org/10.1161/01. STR.0000260205.67348.2b

Pohjasvaara, T., Vataja, R., Leppävuori, A., Kaste, M., \& Erkinjuntti, T. (2001). Depression is an independent predictor of poor long-term functional outcome post-stroke. European Journal of Neurology, 8, 315-319. http://dx.doi. org/10.1046/j.1468-1331.2001.00182.x

Poynter, B., Shuman, M., Diaz-Granados, N., Kapral, M., Grace, S. L., \& Stewart, D. E. (2009). Sex differences in the prevalence of post-stroke depression: A systematic review. Psychosomatics, 50, 563-569.

Roger, V., Go, A., Lloyd-Jones, D., Benjamin, E., Berry, J., Borden, W., . . . Turner, M. (2012). Heart disease and stroke statistics-2012 update: A report from the American Heart Association. Circulation, 125, e2-e220. http:// dx.doi.org/10.1161/CIR.0b013e318245fa41

Snaphaan, L., van der Werf, S., Kanselaar, K., \& de Leeuw, F. E. (2009). Post-stroke depressive symptoms are associated with post-stroke characteristics. Cerebrovascular Diseases, 28, 551-557. http://dx.doi.org/10.1159/ 000247598

Sprinkle, S., Lurie, D., Insko, S., Atkinson, G., Jones, G., Logan, A., \& Bissada, N. (2002). Criterion validity, severity of cut scores, and test-retest reliability of the Beck Depression Inventory-II in a university counseling center sample. Journal of Counseling Psychology, 49, 381-385. http://dx.doi.org/10.1037/0022-0167.49.3.381 